\author{
E. V. Asadova*, V. A. Kofanov** \\ * Oles Gonchar Dnipro National University , \\ ** Oles Gonchar Dnipro National University, \\ Dnipro, 49115. E-mail: vladimir.kofanov@gmail.com
}

\title{
The Bojanov-Naidenov problem for trigonometric polynomials and periodic splines
}

Розглядається задача Боянова-Найденова на множинах $T_{n}$ (тригонометричних поліномів порядку не більшого за $n, n \in \mathbf{N}$ ) та $S_{n, r}$ (періодичних сплайнів порядку $r$, $r \in \mathbf{N}$, мінімального дефекту з вузлами в точках $k \pi / n, k \in \mathbf{Z})$. А саме, для заданих $n, r \in \mathbf{N} ; p, A>0$ i довільного фіксованого відрізку $[a, b] \subset \mathbf{R}$ розв'язана екстремальна задача

$$
\int_{a}^{b}|x(t)|^{q} d t \rightarrow \sup , \quad q \geq p
$$

на класах

$$
T_{n}^{p}(A):=\left\{T \in T_{n}:\|T\|_{p, \delta} \leq A\|\sin n(\cdot)\|_{p, \delta}, \quad \delta \in(0, \pi / n]\right\}
$$

та

$$
\tilde{S}_{n, r}^{p}(A):=\left\{s(\cdot+\tau): s \in S_{n, r},\|s\|_{p, \delta} \leq A\left\|\varphi_{n, r}\right\|_{p, \delta}, \quad \delta \in(0, \pi / n], \quad \tau \in \mathbf{R}\right\}
$$

де

$$
\|x\|_{p, \delta}:=\sup \left\{\|x\|_{L_{p}[a, b]}: \quad a, b \in \mathbf{R}, \quad 0<b-a \leq \delta\right\},
$$

а $\varphi_{n, r}-(2 \pi / n)$-періодичний сплайн Ейлера порядку $r$. Як наслідок, для $k=1, \ldots, r-1$ розв'язана екстремальна задача

$$
\int_{a}^{b}\left|x^{(k)}(t)\right|^{q} d t \rightarrow \sup , \quad q \geq 1
$$

на класах $T_{n}^{p}(A)$ та $\tilde{S}_{n, r}^{p}(A)$.

Доведено, що класи $T_{n}^{p}(A)$ та $\tilde{S}_{n, r}^{p}(A)$ є ширшими за класи

$$
T_{n}(A, p):=\left\{T \in T_{n}: L(T)_{p} \leq A L(\sin n(\cdot))_{p}\right\} .
$$

та

$$
S_{n, r}(A, p):=\left\{s(\cdot+\tau): s \in S_{n, r}, L(s)_{p} \leq A L\left(\varphi_{n, r}\right)_{p}, \tau \in \mathbf{R}\right\},
$$

відповідно, на яких задачі (1) і (2) було розв'язано раніше, де

$$
L(x)_{p}:=\sup \left\{\|x\|_{L_{p}[a, b]}: a, b \in \mathbf{R},|x(t)|>0, t \in(a, b)\right\} .
$$

Крім того, для довільного відрізку $[a, b] \subset \mathbf{R}$ на класах $T_{n}^{p}(A)$ та $\tilde{S}_{n, r}^{p}(A)$ отримано розв'язок відомої задачі Ердеша про характеризацію тригонометричного полігому 
$T \in T_{n}^{p}(A)$ (поліноміального сплайну $s \in \tilde{S}_{n, r}^{p}(A)$ ), графік якого на заданому відрізку має максимальну довжину.

Ключові слова: Задача Болнова-Найденова, поліном, сплайн, перестановка, теорема порівняння.

Для заданих $n, r \in \mathbf{N} ; p, A>0$ и произвольного фиксированного отрезка $[a, b] \subset \mathbf{R}$ решена экстремальная задача $\int_{a}^{b}|x(t)|^{q} d t \rightarrow \sup , q \geq p$, на множествах тригонометрических полиномов $T$ порядка $\leq n$ и $2 \pi$-периодических сплайнов $s$ порядка $r$ минимального дефекта с узлами в точках $k \pi / n, k \in \mathbf{Z}$, удовлетворяющих условию $\|T\|_{p, \delta} \leq A\|\sin n(\cdot)\|_{p, \delta},\|s\|_{p, \delta} \leq A\left\|\varphi_{n, r}\right\|_{p, \delta}, \delta \in(0, \pi / n]$, где $\|x\|_{p, \delta}:=\sup \left\{\|x\|_{L_{p}[a, b]}\right.$ : $a, b \in \mathbf{R}, \quad 0<b-a \leq \delta\}, \mathbf{a} \varphi_{n, r}-(2 \pi / n)$-периодический сплайн Ейлера порядка $r$. Как следствие, решена та же экстремальная задача для промежуточных производных $x^{(k)}, k=1, \ldots, r-1$, при $q \geq 1$.

Ключевые слова: Задача Боянова-Найденова, полином, спдайн, перестановка, теорема сравнения.

For given $n, r \in \mathbf{N} ; p, A>0$ and any fixed interval $[a, b] \subset \mathbf{R}$ we solve the extremal problem $\int_{a}^{b}|x(t)|^{q} d t \rightarrow \sup , q \geq p$, over sets of trigonometric polynomials $T$ of order $\leq n$ and $2 \pi$-periodic splines $s$ of order $r$ and minimal defect with knots at the points $k \pi / n, k \in \mathbf{Z}$, such that $\|T\|_{p, \delta} \leq A\|\sin n(\cdot)\|_{p, \delta},\|s\|_{p, \delta} \leq A\left\|\varphi_{n, r}\right\|_{p, \delta}, \delta \in(0, \pi / n]$, where $\|x\|_{p, \delta}:=\sup \left\{\|x\|_{L_{p}[a, b]}: \quad a, b \in \mathbf{R}, \quad 0<b-a \leq \delta\right\}$ and $\varphi_{n, r}$ is the $(2 \pi / n)$-periodic spline of Euler of order $r$. In particular, we solve the same problem for the intermediate derivatives $x^{(k)}, k=1, \ldots, r-1$, with $q \geq 1$.

Key words: Bojanov-Naidenov problem, polynomial, spline, rearragement, comparison theorem.

MSC 2010: Pri 41A17, Sec 41A44

1. Introduction. Let $G$ denote the real line $\mathbf{R}$ or a finite interval $[a, b]$ or the unite circle $I_{2 \pi}$ wich is realized as the interval $[0,2 \pi]$ with coinsident endpoints. We shall concider the spaces $L_{p}(G), 0<p \leq \infty$, of all measurable functions $x: G \rightarrow \mathbf{R}$ such that $\|x\|_{p}=\|x\|_{L_{p}(G)}<\infty$, where

$$
\begin{gathered}
\|x\|_{p}:=\left(\int_{G}|x(t)|^{p} d t\right)^{1 / p}, \quad \text { if } \quad 0<p<\infty, \\
\|x\|_{p}:=\text { vrai } \sup _{t \in G}|x(t)|, \quad \text { if } \quad p=\infty .
\end{gathered}
$$

For $r \in \mathbf{N}$ and $p, s \in(0, \infty]$ let $L_{p, s}^{r}$ be the space of all functions $x \in L_{p}(\mathbf{R})$ for which $x^{(r-1)}$ is locally absolutely continuous and $x^{(r)} \in L_{s}(\mathbf{R})$. We shall write $\|x\|_{p}$ instead of $\|x\|_{L_{p}(\mathbf{R})}$ and $L_{\infty}^{r}$ instead of $L_{\infty, \infty}^{r}$.

It is well known (see for example [1, page 47]) that the problem of finding the best constant $C$ in the Kolmogorov-Nagy type inequality

$$
\left\|x^{(k)}\right\|_{q} \leq C\|x\|_{p}^{\alpha}\left\|x^{(r)}\right\|_{s}^{1-\alpha}
$$

over the class of functions $x \in L_{p, s}^{r}$, where $\alpha=\frac{r-k+1 / q-1 / s}{r+1 / p-1 / s}, q, p, s \geq 1, r \in \mathbf{N}, k \in \mathbf{N}_{0}:=$ $\mathbf{N} \bigcup\{0\}, k<r, \alpha \leq(r-k) / r$, is equivalently reduced to the following extremal problem:

$$
\left\|x^{(k)}\right\|_{q} \rightarrow \sup
$$


over the class of functions $x \in L_{p, s}^{r}$ satisfying

$$
\left\|x^{(r)}\right\|_{s} \leq A_{r}, \quad\|x\|_{p} \leq A_{0}
$$

where $A_{0}, A_{r}$ are the fix positive numbers.

Many mathematicians investigated the problem of finding the best constant in (1.1). There are only few cases in which sharp inequalities of the form (1.1) are known for any $r \in \mathbf{N}$ and any $k<r$. The survey of the results in this directions are given in [1] - [3]. For arbitrary interval $[a, b] \subset \mathbf{R}$ Bojanov and Naidenov have solved the problem

$$
\int_{a}^{b} \Phi\left(\left|x^{(k)}(t)\right|\right) d t \rightarrow \sup , \quad k=1, \ldots r-1,
$$

over the class of functions $x \in L_{\infty}^{r}$ satisfying (1.3) with $p=s=\infty$, where $\Phi$ is continuously differentiable function on $[0, \infty)$, positive on $(0, \infty)$ and such that $\Phi(t) / t$ is non-decreasing and $\Phi(0)=0$. In particularly, Bojanov and Naidenov have solved the problem of Erdös [5] on characterization of the trigonometric polynomial of fixed uniform norm that has maximal arc length over $[a, b]$.

We shall consider the class $W$ of the continuous, nonnegative and convex functions $\Phi$ definsd on $[0, \infty)$ and such that $\Phi(0)=0$. For $p>0$ set $[6]$

$$
L(x)_{p}:=\sup \left\{\|x\|_{L_{p}[a, b]}: a, b \in \mathbf{R},|x(t)|>0, t \in(a, b)\right\} .
$$

Note that $L(x)_{\infty}=\|x\|_{\infty}$ and $L\left(x^{\prime}\right)_{1} \leq 2\|x\|_{\infty}$.

The following modification of the Bojanov and Naidenov problem was solved in [7]:

$$
\int_{a}^{b} \Phi\left(|x(t)|^{p}\right) d t \rightarrow \sup , \quad \Phi \in W, \quad p>0,
$$

over the class of functions $x \in L_{\infty}^{r}$ satisfying

$$
\left\|x^{(r)}\right\|_{\infty} \leq A_{r}, \quad L(x)_{p} \leq A_{0}
$$

As a special case was solved the problem

$$
\int_{a}^{b} \Phi\left(\left|x^{(k)}(t)\right|\right) d t \rightarrow \sup , \quad \Phi \in W, \quad k=1, \ldots, r-1,
$$

over the same class of functions $x \in L_{\infty}^{r}$.

The generalizations of the results of the article [7] are given in [8], [9].

Denote by $\varphi_{r}(t), r \in \mathbf{N}$, the $r^{t h} 2 \pi$-periodic integral with zero mean value on a period of the function $\varphi_{0}(t)=\operatorname{sgn} \sin t$ and for $\lambda>0$ set $\varphi_{\lambda, r}(t):=\lambda^{-r} \varphi_{r}(\lambda t)$. 
The solution of the problems (1.5) and (1.7) was given in [10] over the class of functions $x \in L_{\infty}^{r}$ satisfying

$$
\left\|x^{(r)}\right\|_{\infty} \leq A_{r}, \quad\|x\|_{p, \delta} \leq A_{r} \cdot\left\|\varphi_{\lambda, r}\right\|_{p, \delta}, \quad \delta \in(0, \pi / \lambda]
$$

where

$$
\|x\|_{p, \delta}:=\sup \left\{\|x\|_{L_{p}[a, b]}: a, b \in \mathbf{R}, \quad 0<b-a \leq \delta\right\} .
$$

Note that the value $\|x\|_{p, \delta}$ for $p \geq 1$ is the norm but the value $L(x)_{p}$ is not.

Let $n, r \in \mathbf{N}$ and $p, A>0$. Denote by $T_{n}$ the set of all trigonometric polynomials of order $\leq n$. Let $S_{n, r}$ be the set of $2 \pi$-periodic polynomial splines of order $r$ with knots at the points $k \pi / n, k \in \mathbf{Z}$. Set

$$
T_{n}^{p}(A):=\left\{T \in T_{n}:\|T\|_{p, \delta} \leq A\|\sin n(\cdot)\|_{p, \delta}, \quad \delta \in(0, \pi / n]\right\}
$$

and

$$
S_{n, r}^{p}(A):=\left\{s \in S_{n, r}:\|s\|_{p, \delta} \leq A\left\|\varphi_{n, r}\right\|_{p, \delta}, \quad \delta \in(0, \pi / n]\right\}
$$

where the value $\|x\|_{p, \delta}$ is defined by (1.8).

We solve in this paper the problem (1.5) and (1.7) over the classes $T_{n}^{p}(A)$ and $S_{n, r}^{p}(A)$ (Theorems 1 and 3 ). As a special case we solve the problem Erdös over the same classes.

2. Preliminaries. For $n, r \in \mathbf{N}$ and $p, A>0$ set

$$
\begin{gathered}
\psi_{n, r}(t)=\psi_{n, r}(K, t):=A \sin n t, \quad \text { if } \quad K=T_{n}^{p}(A), \\
\psi_{n, r}(t)=\psi_{n, r}(K, t):=A \varphi_{n, r}(t), \quad \text { if } \quad K=S_{n, r}^{p}(A) .
\end{gathered}
$$

Lemma 1. If a function $x$ is continuous on $\mathbf{R}$ and the supremum

$$
\sup \left\{\int_{\alpha}^{\beta}|x(t)| d t: \quad \alpha, \beta \in \mathbf{R}, \quad \beta-\alpha \leq \delta\right\}
$$

is realized on interval $[a, b]$ then $|x(a)|=|x(b)|[11]$.

Lemma 2. Let $n, r \in \mathbf{N}$ and $p, A>0$. If $K=T_{n}^{p}(A)$ or $K=S_{n, r}^{p}(A)$ then, for any function $x \in K$, the following inequality holds true

$$
\|x\|_{\infty} \leq\left\|\psi_{n, r}(K, \cdot)\right\|_{\infty}
$$

Proof. Fix a function $x \in K$ and arbitrary $a \in \mathbf{R}$. Let $m$ be a point of maximum $\psi_{n, r}(K, t)$. Note that

$$
\left\|\psi_{n, r}(K, \cdot)\right\|_{p, \delta}^{p}=\int_{m-\delta / 2}^{m+\delta / 2} \psi_{n, r}^{p}(K, t) d t, \quad \delta \in(0, \pi / n] .
$$


So we conclude from (1.9), (1.10) and (2.1) that

$$
\frac{1}{\delta} \int_{a}^{a+\delta}|x(t)|^{p} d t \leq \frac{1}{\delta} \int_{m-\delta / 2}^{m+\delta / 2} \psi_{n, r}^{p}(K, t) d t=\frac{2}{\delta} \int_{m}^{m+\delta / 2} \psi_{n, r}^{p}(K, t) d t .
$$

Letting $\delta \rightarrow 0$ yields

$$
|x(a)|^{p} \leq \psi_{n, r}^{p}(K, m)=\left\|\psi_{n, r}(K, \cdot)\right\|_{\infty}^{p},
$$

wich is equivalent to $(2.2)$.

Lemma 2 is proved.

We shall call $f \in L_{\infty}^{1}(\mathbf{R})$ is comparison function for $x \in L_{\infty}^{1}(\mathbf{R})$ if $\|x\|_{\infty} \leq\|f\|_{\infty}$ and it follows from $x(\xi)=f(\eta), \xi, \eta \in \mathbf{R}$, the inequality $\left|x^{\prime}(\xi)\right| \leq\left|f^{\prime}(\eta)\right|$ (if there are the derivatives).

Lemma 3. Let $n, r \in \mathbf{N}$ and $p, A>0$. If $K=T_{n}^{p}(A)$ or $K=S_{n, r}^{p}(A)$ then the function $\psi_{n, r}(K, t)$ is the comparison function for any function $x \in K$.

Proof. Fix a function $x \in K$. By Lemma 2 the inequality (2.2) holds true. If $K=T_{n}^{p}(A)$ then it follows from (2.2) (see, for example, the proof of Theorem 8.1.1 [1]) that the function $\psi_{n, r}(K, t)=A \sin n t$ is the comparison function for $x$.

Let now $K=S_{n, r}^{p}(A)$. Then $\psi_{n, r}(K, t)=A \varphi_{n, r}(t)$. So applying Tikhomirov inequality (see, for example, [1, Lemma 8.2.1])

$$
\left\|x^{(r)}\right\|_{\infty} \leq \frac{\|x\|_{\infty}}{\left\|\varphi_{n, r}\right\|_{\infty}}, \quad x \in S_{n, r}
$$

we conlude from (2.2) that $\left\|x^{(r)}\right\|_{\infty} \leq A$. Hence, in view of $(2.2)$ the function $x$ satiesfies the conditions of Kolmogorov comparison Theorem [13]. By this Theorem the function $\psi_{n, r}(K, t)=A \varphi_{n, r}(t)$ is the comparison function for $x$.

Lemma 3 is proved.

Let $x \in L_{1}[a, b]$. The rearrangment of the function $|x|$ is denoted by $r(x, t)$ (see, for example, $[14, \S 1.3])$. We also set $r(x, t)=0$ for $t>b-a$.

Lemma 4. Let $n, r \in \mathbf{N} ; p, A>0 ; \Phi \in W$. If $K=T_{n}^{p}(A)$ or $K=S_{n, r}^{p}(A)$ then, for any function $x \in K$ and arbitrary interval $[a, b] \subset \mathbf{R}$ with $b-a \leq \pi / n$ there holds the inequality

$$
\int_{a}^{b} \Phi\left(|x(t)|^{p}\right) d t \leq \int_{m-\Theta}^{m+\Theta} \Phi\left(\left|\psi_{n, r}(K, t)\right|^{p}\right) d t
$$

where $m$ is a point of maximum of the function $\psi_{n, r}(K, t)$ and the number $\Theta$ is such that

$$
\psi_{n, r}(m-\Theta)=\psi_{n, r}(m+\Theta), \quad 2 \Theta=b-a .
$$


In particularly,

$$
\int_{a}^{b} \Phi\left(|x(t)|^{p}\right) d t \leq \int_{c}^{c+\pi / n} \Phi\left(\left|\psi_{n, r}(K, t)\right|^{p}\right) d t,
$$

where $c$ is a zero of the function $\psi_{n, r}(K, t)$.

Proof. Fix a function $x \in K$ and interval $[a, b]$ satiesfying conditions of Lemma 4 . Let us prove (2.3). Set $\delta:=b-a$ and let the supremum

$$
\sup \left\{\int_{\alpha}^{\beta} \Phi\left(|x(t)|^{p}\right) d t: \quad \alpha, \beta \in \mathbf{R}, \quad \beta-\alpha \leq \delta\right\}
$$

is realized on an interval $[\alpha, \beta]$. Obviously, there is such interval that $\beta-\alpha=\delta$. It is enough to prove the inequality $(2.3)$ for $[a, b]=[\alpha, \beta]$. Then, by Lemma 1

$$
|x(a)|=|x(b)|
$$

Denote by $\bar{x}$ the restriction of the function $x$ on $[a, b]$ and let $\bar{\psi}$ be the restriction of the function $\psi_{n, r}$ on $[m-\Theta, m+\Theta]$. Let us prove the inequality

$$
\int_{0}^{\xi} r^{p}(\bar{x}, t) d t \leq \int_{0}^{\xi} r^{p}(\bar{\psi}, t) d t, \quad \xi>0 .
$$

First, we show that the difference $\delta(t):=r(\bar{x}, t)-r(\bar{\psi}, t)$ has at most one change of sign on $[0, \infty)$ (from - to + ). Note that

$$
\delta(0) \leq\|x\|_{\infty}-\left\|\psi_{n, r}\right\|_{\infty} \leq 0
$$

in view of Lemma 2. Set

$$
A:=\min \{|\bar{x}(t)|: t \in[a, b]\}, \quad B:=\max \{|\bar{x}(t)|: t \in[a, b]\} .
$$

If $B \leq\left|\psi_{n, r}(m+\Theta)\right|$ then the difference $\delta(t)$ has no change of sign. Assume that $B>\left|\psi_{n, r}(m+\Theta)\right|$ and set $C=\max \left\{A,\left|\psi_{n, r}(m+\Theta)\right|\right\}$. Because of $(2.5)$ and (2.7), for any $z \in(C, B)$, there exists the points

$$
t_{i} \in[a, b], \quad i=1, \ldots, m, \quad m \geq 2, \quad y_{j} \in[m-\Theta, m+\Theta], \quad j=1,2,
$$

such that

$$
z=\left|\bar{x}\left(t_{i}\right)\right|=\left|\bar{\psi}\left(y_{j}\right)\right| .
$$

By Lemma $3 \psi_{n, r}$ is the comparison function for $x$. So for the points $t_{i}$ and $y_{j}$, satisfying (2.8), the following inequality holds true

$$
\left|\bar{x}^{\prime}\left(t_{i}\right)\right| \leq\left|\bar{\psi}^{\prime}\left(y_{j}\right)\right|
$$


Hence, if the points $\Theta_{1}, \Theta_{2}>0$ are chosen such that

$$
z=r\left(\bar{x}, \Theta_{1}\right)=r\left(\bar{\psi}, \Theta_{2}\right)
$$

then by the theorem about differentiation of rearrengement (see, for example, [14, statement 1.3.2])

$$
\left|r^{\prime}\left(\bar{x}, \theta_{1}\right)\right|=\left[\sum_{i=1}^{m}\left|\bar{x}^{\prime}\left(t_{i}\right)\right|^{-1}\right]^{-1} \leq\left[\sum_{j=1}^{2}\left|\bar{\psi}^{\prime}\left(y_{j}\right)\right|^{-1}\right]^{-1}=\left|r^{\prime}\left(\bar{\psi}, \theta_{2}\right)\right| .
$$

It follows that the difference $\delta(t):=r(\bar{x}, t)-r(\bar{\psi}, t)$ has at most one change of sign on $[0, \infty)$ (from - to + ). It is exactly the same for difference $\delta_{p}(t):=r^{p}(\bar{x}, t)-r^{p}(\bar{\psi}, t)$. Let us consider the integral

$$
I_{p}(\xi):=\int_{0}^{\xi} \delta_{p}(t) d t
$$

It is clear that $I_{p}(0)=0$. Taking into account the definitions (1.9) and (1.10) of the classes $T_{n}^{p}(A)$ and $S_{n, r}^{p}(A)$ we have

$$
I_{p}(\xi)=\|x\|_{p, \delta}-\left\|\psi_{n, r}\right\|_{p, \delta} \leq 0, \quad \xi \geq \delta .
$$

Besides, the derivative $I_{p}^{\prime}(t)=\delta_{p}(t)$ has at most one change of sign on $[0, \infty)$ (from - to + ). Therefore, $I_{p}(\xi) \leq 0$ for all $\xi \geq 0$ and the inequality (2.6) is proved. Applying Theorem of Hardy-Littelwood-Polya (see, for example, [14, Statement 1.3.11]) we deduce (2.3) from (2.6). It is evident that (2.4) follows from (2.3).

Lemma 3 is proved.

Corollary 1. Let $n, r \in \mathbf{N} ; p, A>0$. Then, for any $q>p$, we have

$$
T_{n}^{p}(A) \subset T_{n}^{q}(A) ; \quad S_{n, r}^{p}(A) \subset S_{n, r}^{q}(A),
$$

where the classes $T_{n}^{p}(A)$ and $S_{n, r}^{p}(A)$ are defined by (1.9) and (1.10).

Proof. Setting $\Phi(t)=t^{q / p}$ in the inequality (2.3) and taking into account the definitions (1.9) and (1.10) we immediately derive both inclusions.

3. The main results. Let $n, r \in \mathbf{N} ; p, A>0 ;[a, b] \subset \mathbf{R}$. Following Bojanov and Naidenov [4] let us write the length of $[a, b]$ in the form

$$
b-a=l \cdot \frac{\pi}{n}+2 \Theta, \quad l \in \mathbf{N} \bigcup\{0\}, \quad 2 \Theta \in[0, \pi / n) .
$$

Choose $\tau \in \mathbf{R}$ such that

$$
\left|\psi_{n, r}(a+\Theta+\tau)\right|=\left|\psi_{n, r}(b-\Theta+\tau)\right|=\left\|\psi_{n, r}\right\|_{\infty},
$$

where the function $\psi_{n, r}$ is defined by (2.1). Set

$$
\tilde{S}_{n, r}^{p}(A):=\left\{s(\cdot+\tau): s \in S_{n, r}^{p}(A), \quad \tau \in \mathbf{R}\right\}
$$

(the definition of the class $S_{n, r}^{p}(A)$ is given by (1.10)). If $K=T_{n}^{p}(A)$ (see (1.9)) or $K=\tilde{S}_{n, r}^{p}(A)$ then it is clear that $\psi_{n, r}(\cdot+\tau) \in K$ for any $\tau \in \mathbf{R}$. 
Theorem 1. Let $n, r \in \mathbf{N} ; p, A>0$. If $K=T_{n}^{p}(A)$ or $K=\tilde{S}_{n, r}^{p}(A)$ then, for any function $\Phi \in W$ and arbitrary interval $[a, b] \subset \mathbf{R}$,

$$
\sup \left\{\int_{a}^{b} \Phi\left(|x(t)|^{p}\right) d t: \quad x \in K\right\}=\int_{a}^{b} \Phi\left(\left|\psi_{n, r}(t+\tau)\right|^{p}\right) d t,
$$

where the number $\tau$ is defined by (3.2). In particularly, for $q \geq p$,

$$
\sup \left\{\int_{a}^{b}|x(t)|^{q} d t: \quad x \in K\right\}=\int_{a}^{b}\left|\psi_{n, r}(t+\tau)\right|^{q} d t .
$$

Proof. Fix any function $x \in K$ and arbitrary interval $[a, b] \subset \mathbf{R}$. Let us write the length of $[a, b]$ in the form (3.1). Set $a_{k}:=a+k \pi / n, k=0,1, \ldots, l$. By Lemma 4

$$
\int_{a_{k}}^{a_{k+1}} \Phi\left(|x(t)|^{p}\right) d t \leq \int_{c}^{c+\pi / n} \Phi\left(\left|\psi_{n, r}(t)\right|^{p}\right) d t, \quad k=0,1, \ldots, l-1,
$$

and

$$
\int_{a_{l}}^{b} \Phi\left(|x(t)|^{p}\right) d t \leq \int_{m-\Theta}^{m+\Theta} \Phi\left(\left|\psi_{n, r}(t)\right|^{p}\right) d t
$$

where $c$ is a zero, $m$ is a point of maximum of the function $\psi_{n, r}$ and the number $\Theta$ is defined by (3.1). Therefore,

$$
\begin{gathered}
\int_{a}^{b} \Phi\left(|x(t)|^{p}\right) d t \leq l \cdot \int_{c}^{c+\pi / n} \Phi\left(\left|\psi_{n, r}(t)\right|^{p}\right) d t+\int_{m-\Theta}^{m+\Theta} \Phi\left(\left|\psi_{n, r}(t)\right|^{p}\right) d t= \\
=\int_{a}^{b} \Phi\left(\left|\psi_{n, r}(t+\tau)\right|^{p}\right) d t .
\end{gathered}
$$

Moreover, the equality is realized here for the function $x(t)=\psi_{n, r}(t+\tau)$. First statement of Theorem 1 is proved. Setting $\Phi(t)=t^{q / p}$ in it we have second statement.

Theorem 1 is proved.

Foe $n, r \in \mathbf{N}$ and $A, p>0$ set

$$
T_{n}(A, p):=\left\{T \in T_{n}: L(T)_{p} \leq A L(\sin n(\cdot))_{p}\right\} .
$$

and

$$
S_{n, r}(A, p):=\left\{s(\cdot+\tau): s \in S_{n, r}, L(s)_{p} \leq A L\left(\varphi_{n, r}\right)_{p}, \tau \in \mathbf{R}\right\},
$$

where the value $L(x)_{p}$ is defined by (1.4). For $K=T_{n}(A, p)$ or $K=S_{n, r}(A, p)$ define the function

$$
\begin{array}{ccc}
\psi_{n, r}(t)=\psi_{n, r}(K, t):=A \sin n t, & \text { if } \quad K=T_{n}(A, p), \\
\psi_{n, r}(t)=\psi_{n, r}(K, t):=A \varphi_{n, r}(t), & \text { if } \quad K=S_{n, r}(A, p) .
\end{array}
$$


Theorem 2. Let $n, r \in \mathbf{N} ; p, A>0$. Then

$$
T_{n}(A, p) \subset T_{n}^{p}(A), \quad S_{n, r}(A, p) \subset \tilde{S}_{n, r}^{p}(A),
$$

wrere the classes $T_{n}^{p}(A)$ and $\tilde{S}_{n, r}^{p}(A)$ is defined by (1.9) and (3.3) .

Proof. Fix any function $x \in K$ ( where $K=T_{n}(A, p)$ or $K=S_{n, r}(A, p)$ ) and the number $\delta \in(0, \pi / n]$. Let us prove the inequality

$$
\|x\|_{p, \delta} \leq\left\|\psi_{n, r}\right\|_{p, \delta}
$$

For a function $x \in T_{n}(A, p)$ or $x \in S_{n, r}(A, p)$ and arbitrary interval $[a, b]$ satisfying $b-a \leq \pi / n$ it was proved $[9$, теоремы 7,9$]$ that

$$
\int_{a}^{b} \Phi\left(|x(t)|^{p}\right) d t \leq \int_{m-\Theta}^{m+\Theta} \Phi\left(\left|\psi_{n, r}(t)\right|^{p}\right) d t, \quad \Phi \in W
$$

where $m$ is a point of maximum of the function $\psi_{n, r}$ and $2 \Theta=b-a$. Setting $\Phi(t)=t$ in the inequality (3.6) we have the estimate (3.5). It follows from (3.5) in view of the definitions (1.9), (1.10) and (3.3) both of inclusions.

Theorem 2 is proved.

Remark 1. It follows from Theorem 2 that the problem (1.5) is proved in Theorem 1 over wider classes than in Theorems 7 and 9 in [9] where this problem has been proved over the classes $T_{n}(A, p)$ and $\left.S_{n, r}(A, p)\right)$.

Next Theorem contains a solution of the problem (1.7) over this more wide classes $T_{n}^{p}(A)$ and $\left.\tilde{S}_{n, r}^{p}(A)\right)$.

Let $n, k, r \in \mathbf{N} ; A, p>0 ;[a, b] \subset \mathbf{R}$ and the length of the interval $[a, b]$ is presented in the fom (3.1). Choose $\tau_{k} \in \mathbf{R}$ such that

$$
\left|\psi_{n, r}^{(k)}\left(a+\Theta+\tau_{k}\right)=\right| \psi_{n, r}^{(k)}\left(b-\Theta+\tau_{k}\right) \mid=\left\|\psi_{n, r}^{(k)}\right\|_{\infty},
$$

where the function $\psi_{n, r}(K, t)$ is defined by (2.1). Besides, let $k \leq r$ for $\left.K=\tilde{S}_{n, r}^{p}(A)\right)$.

Theorem 3. Let $n, k, r \in \mathbf{N} ; A, p>0$. If $K=T_{n}^{p}(A)$ or $K=\tilde{S}_{n, r}^{p}(A)$ and $k \leq r$ then, for any function $\Phi \in W$ and arbitrary interval $[a, b] \subset \mathbf{R}$, we have

$$
\sup \left\{\int_{a}^{b} \Phi\left(\left|x^{(k)}(t)\right|\right) d t: \quad x \in K\right\}=\int_{a}^{b} \Phi\left(\left|\psi_{n, r}^{(k)}\left(t+\tau_{k}\right)\right|\right) d t
$$

where the number $\tau_{k}$ is define by (3.7). In particularly, for any $q \geq 1$,

$$
\sup \left\{\int_{a}^{b}\left|x^{(k)}(t)\right|^{q} d t: \quad x \in K\right\}=\int_{a}^{b}\left|\psi_{n, r}^{(k)}\left(t+\tau_{k}\right)\right|^{q} d t .
$$


Proof. Fix any function $x \in K$ and arbitrary interval $[a, b] \subset \mathbf{R}$. Let us prove the first statement of Theorem 3. By Lemma 2

$$
\|x\|_{\infty} \leq\left\|\psi_{n, r}\right\|_{\infty}
$$

It follows that

$$
\left\|x^{(i)}\right\|_{\infty} \leq\left\|\psi_{n, r}^{(i)}\right\|_{\infty}
$$

where $i \in \mathbf{N}$ if $K=T_{n}^{p}(A)$ and $i=1,2, \ldots, r$ if $K=\tilde{S}_{n, r}^{p}(A)$. Really, $\psi_{n, r}(t)=A \sin n t$ if $K=T_{n}^{p}(A)$ and the inequality (3.9) follows from (3.8) and well known Bernstein inequality

$$
\left\|x^{(i)}\right\|_{\infty} \leq n^{i} \cdot\|x\|_{\infty}, \quad i \in \mathbf{N}
$$

for trigonometric polynomials $x \in T_{n}$. If $K=\tilde{S}_{n, r}^{p}(A)$ then $\psi_{n, r}(t)=A \varphi_{n, r}(t)$ and $(3.9)$ follows from (3.8) and Tikhomirov inequality (see, for example, [1, Theorem 8.2.1])

$$
\left\|x^{(i)}\right\|_{\infty} \leq \frac{\left\|\varphi_{n, r-i}\right\|_{\infty}}{\left\|\varphi_{n, r}\right\|_{\infty}} \cdot\|x\|_{\infty}, \quad i=1,2, \ldots, r
$$

for splines $x \in S_{n, r}$. So for any interval $[\alpha, \beta]$ satisfying $\left|x^{(k)}(t)\right|>0, \quad t \in(\alpha, \beta)$, we have

$$
\int_{\alpha}^{\beta}\left|x^{(k)}(t)\right| d t=\left|x^{(k-1)}(\beta)-x^{(k-1)}(\alpha)\right| \leq 2\left\|x^{(k-1)}\right\|_{\infty} \leq 2\left\|\psi_{n, r}^{(k-1)}\right\|_{\infty}=L\left(\psi_{n, r}^{(k)}\right)_{1},
$$

where the value $L(x)_{p}$ is defined by (1.4). It follows that $L\left(x^{(k)}\right)_{1} \leq L\left(\psi_{n, r}^{(k)}\right)_{1}$. For a function $x \in T_{n}$ or $x \in S_{n, r}$ satisfying last inequality and for arbitrary innterval $[a, b]$ it was proved $[9$, теоремы 7,9$]$ the estimate

$$
\int_{a}^{b} \Phi\left(\left|x^{(k)}(t)\right|\right) d t \leq \int_{a}^{b} \Phi\left(\left|\psi_{n, r}^{(k)}\left(t+\tau_{k}\right)\right|\right) d t, \quad \Phi \in W
$$

where $\tau_{k}$ is defined by (3.7). The equality is odtained here for the function $x(t)=$ $\psi_{n, r}\left(t+\tau_{k}\right)$. The first statement of Theorem 3 is proved. Setting in it $\Phi(t)=t^{q}$ we have the second statement. Theorem 3 is proved.

It is well known that arc length $l[a, b]$ over $[a, b]$ of a function $x \in L^{1}[a, b]$ is given by formula $l[a, b]=\int_{a}^{b} \sqrt{1+x^{\prime}(t)^{2}} d t$. It is clear that $\Phi_{0} \in W$ for the function $\Phi_{0}(t)=$ $\sqrt{1+t^{2}}$. Consequently, setting $\Phi=\Phi_{0}, k=1$ in the first statement of Theorem 3 we obtain the solution of the problem of Erdös on characterisation of trigonometric polynomials $T \in T_{n}^{p}(A)$ that has maximal arc length over $[a, b]$. Besides, we solve the same problem over the space of splines $\tilde{S}_{n, r}^{p}(A)$.

Corollary 2. Let $n, r \in \mathbf{N} ; A, p>0 ;[a, b] \subset \mathbf{R}$.

Among all trigonometric polynomials $T \in T_{n}^{p}(A)$ the maximal arc length over $[a, b]$ has the polynomial $T=A \sin n\left(t+\tau_{1}\right)$, where $\tau_{1}$ is the same as in Theorem 3.

Among all splines $s \in \tilde{S}_{n, r}^{p}(A)$ the maximal arc length over $[a, b]$ has the spline $A \varphi_{n, r}\left(t+\tau_{1}\right)$, where $\tau_{1}$ is the same as in Theorem 3. 


\section{References}

1. Корнейчук H. П. Неравенства для производных и их приложения [Текст]/ Н. П. Корнейчук, В. Ф. Бабенко, В. А. Кофанов, С. А. Пичугов// К.: Наукова думка, 2003. - 590 c.

2. Бабенко В. Ф. Исследования Днепропетровских математиков по неравенствам для производных периодических функций и их приложениям $[$ Текст]/ В. Ф. Бабенко // Укр. мат. журн. - 2000. - 52, 1. - С. 5 - 29.

3. Kwong M. K. Norm Inequalities for Derivatives and Differences [Text] / M. K. Kwong, A. Zettl [Text]// Berlin: Springer-Verlag, 1992. - 150 p. (Lecture Notes in Math. - V. 1536).

4. Bojanov B. An extension of the Landau-Kolmogorov inequality. Solution of a problem of Erdos [Text] / B. Bojanov, N. Naidenov// Journal d'Analyse Mathematique. - 1999. 78. - P. $263-280$.

5. Erdös P. Open problems// In: Open Problems in Approximation Theory [Text]/ P. Erdös// SCT Publishing, Singapur. - 1994. - P. 238 - 242.

6. Pinkus A. Shisha O., Variations on the Chebyshev and $L^{q}$ Theories of Best Approximation [Text] / A. Pinkus, O. Shisha// Journal of Approximation Theory. - 1982. - 35, 2. - P. 148-168.

7. Кофанов В. А. О некоторых экстремальных задачах разных метрик для дифференцируемых функций на оси [Текст] / В. А. Кофанов // Укр. мат. журн. - 2009. - 61, 6. - C. $765-776$.

8. Kofanov V. A. Some extremal problems various metrics and sharp inequalities of NagyKolmogorov type [Text] / V. A. Kofanov// East. J. Approxim. - 2010. - 16, 4. - C. 313 -334 .

9. Кофанов B. A. Точные верхние грани норм функций и их производных на классах функций с заданной функцией сранения [Текст] /В. А. Кофанов // Укр. мат. журн. - 2011. - 63, 7. - C. $969-984$.

10. Кофанов В. А. Задача Боянова-Найденова для дифференцируемых функций на оси и неравенства разных метрик [Текст] /В. А. Кофанов // Укр. мат. журн.- 2019. - 71, 6. - C. $786-800$.

11. Кофанов В. А. Неравенства разных метрик для дифференцируемых периодических функций $[$ Текст] /В. А. Кофанов // Укр. мат. журн. - 2015. - 67, 2. - С. $202-212$.

12. Колмогоров А. Н. О неравенствах между верхними гранями последовательных производных функции на бесконечном интервале [Текст] / А. Н. Колмогоров // Избр. труды. Математика, механика.- М.: Наука, 1985. - 470 с.- С. 252-263.

13. Корнейчук H. П. Экстремальные свойства полиномов и сплайнов [Текст] / Н. П. Корнейчук, В. Ф. Бабенко, А. А. Лигун// К.: Наукова думка, 1992. - 304 c.

Received: 30.01.2019. Accepted: 10.06.2019 